\title{
Human Recognition System using Cepstral Information
}

\author{
Emna RABHI \\ Département Génie Electrique \\ Université Tunis EL Manar, Ecole Nationale d'Ingénieurs \\ de TUNIS, LR Signal, Image et Technologies de \\ l'information, BP 37, Belvédère, 1002 \\ Tunis, Tunisie \\ emna.rabhi@gmail.com
}

\author{
Zied Lachiri \\ Département Génie physisque et instrumentation \\ Institut National des sciences appliquées et de technologie \\ Tunis, Tunisie \\ zied.lachiri@enit.rnu.tn
}

\begin{abstract}
This paper presents a new method for human recognition using the cepstral information. The proposed method consists in extracting the Linear Frequency Cepstral Coefficients (LFCC) from each heartbeat in the homomorphic domain. Thus, the Hidden Markov Model (HMM) under Hidden Markov Model Toolkit (HTK) is used for electrocardiogram (ECG) classification. To evaluate the performance of the classifier, the number of coefficients and the number of frequency bands are varied. Concerning the HMM topology, the number of Gaussians and states are also varied. The best rate is obtained with 32 coefficients, 24 frequency bands, 1 Gaussian and 5 states. Further, the method is improved by adding dynamic features: the first order delta $(\Delta)$ and energy (E) to the coefficients.

The approach is evaluated on 18 healthy signals of the MIT_BIH database.

The obtained results reveal which LFCC with energy that make a 33 dimensional feature vector leads to the best human recognition rate which is $99.33 \%$.
\end{abstract}

Keywords- Electrocardiogram(ECG); Linear Frequency Cepstral Coefficients( LFCC); Hidden Markov Model (HMM).

\section{INTRODUCTION}

Biometrics is a secure alternative to traditional methods of identity verification of individuals, such as passwords. The increasing need for security leads to the growth of biometrics, and the search for new biometric technology becomes topical. Biometrics uses the physiological or behavioral characteristics that are unique to each one in order to determine the identity of individuals [1]. There are several types of biometric methods such as the fingerprint, hand geometry, face recognition, iris, etc... Unfortunately, with the development of technology falsification, these features can be forged. Hence, the need for new research which could be difficult to imitate is behind the use of the physiological signals ECG as a biometric characteristic [9].

Biel et al. [4] are the earliest researchers who have worked with ECG as a biometric characteristic. They have extracted twelve features from each record for human recognition in the time domain. For classification, the SIMCA (Soft Independent Modeling of Class Analogy) model was used.

Shen et al. [13] have limited their interest in their recognition algorithm, to only a few fiducial points, surrounding the QRS complex. They have extracted seven features. To evaluate the performance of their method, they have used neural network (DBNN) and template matching.

Israel et al. [10] have determined the ECG signal peaks in the time domain by finding local maxima in the regions surrounding each of the P, R and T waves. Then, 15 features were extracted which denote the time distance between detected features.

To sum up, the major shortcomings of the previous works is the following: the method in detection of fiducial points; because no universally acknowledged definition exists for defining exactly where the wave boundaries lie and the physiological changes of the heart.

In this paper, an attempt is made to present a new method based on features extraction of ECG without fiducial detection. To improve the identification accuracy, an approach based on cepstral information is introduced. It is reflected in the compute of the LFCC from each heart beat of the signal ECG.

This paper is organized as follows. Section 2 presents the human Recognition System that contains a description of the features extraction and the classifier HMM. Section 3 is about the experimental results of our method. Finally, conclusions are presented in Section 4.

\section{HUMAN RECOGNITION SYSTEM}

The electrocardiogram is a non-periodic [3] but highly recurrent signal that is why we used the cepstral coefficients with linear filter banks [6,7]. The human recognition system consists in extraction of LFCC of each heart beat which were varied from 12 to 34 . A package named LFCC-RASTAMAT [15] was adapted for extracting LFCC features.

In order to improve the results, dynamic features were added to the coefficients which are: first order delta $(\Delta)$ and energy (E).

In Electrocardiogram classification, Hidden Markov Model (HMM) was used. To choose the best model topology of the classifier, the number of states was varied from 5 to 7 and the number of Gaussians from 1 to 5.

\section{A. Features Extraction}

- Linear Frequency Cepstral Coefficients ( LFCC)

The cepstral coefficients are calculated from the modulus 
of the fast Fourier transform of the signal windowed by Hamming. An analysis filter bank module converts energy by bands coefficients. The scale filter banks used in this work is linear to calculate the Linear Frequency Cepstral Coefficients (LFCC) [14].

The block diagram illustrating the steps of calculating LFCC is as follows:

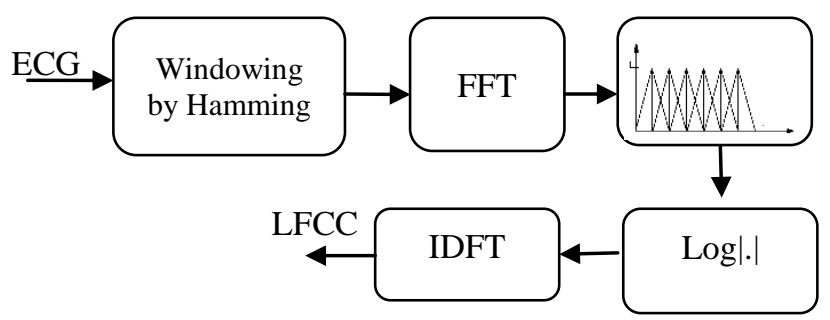

Fig 1. LFCC Algorithm.

\section{- $\quad$ Energy (E)}

In order to identify the variations of an ECG signal, the energy is often evaluated in several successive frames. For a frame " $i$ ", the energy parameter is computed as follows:

$$
E=\sum_{n=0}^{N-1} X_{i}^{2}[n]
$$

\section{- The first order delta $(\Delta)$}

Calculating the first order delta of an ECG signal means computing its first derivative. The first derivative is the rate of change of $y$ with $x$ : dy/dx note that $x$ and $y$ denote the coordinates of signal's points. In other words, it is the slope of the tangent to the signal at each point.

\section{B. Hidden Markov Model (HMM)}

A HMM is a stochastic model. After learning, it is able to provide the probability of generating a given set of observations. This model is defined by a sequence of $\mathrm{N}$ states qt connected by transitions aij at time $t$ [5].

The training of HMM model according to the prototype model is initialized by the Viterbi algorithm, and then reestimated by the Baum-Welch algorithm. The extracted parameters are parameterized vectors whose representation defined the set of observations $\mathrm{O}=\{01,02, \ldots, \mathrm{oT}\}$ which are useful in the process of modeling the HMM.

The Bakis Model is adopted as a HMM topology. It is a model left to right with 5 states whose initial and final states do not emit vectors of observations as shown in Figure2. The mean vector of the used model is initialized to zero and a variance vector is initialized to one.

For building and manipulating the Hidden Markov models, a platform Hidden Markov Model Toolkit (HTK) [11] was used. It is a platform formed by a set of libraries and tools software written in language $\mathrm{C}$, its modularity allows to develop its own software and uses the tools available directly [12].

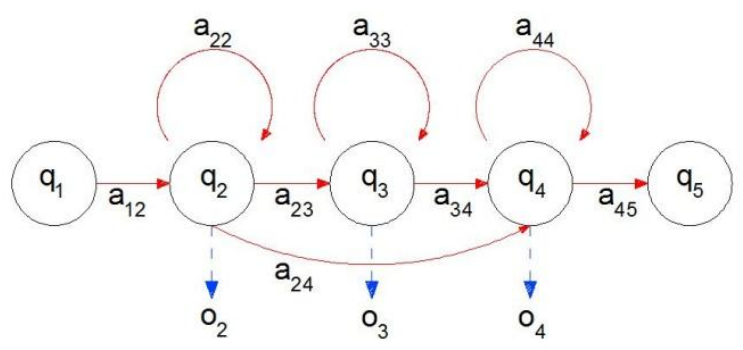

Fig 2. Bakis Model.

\section{- Training}

Recognition of data requires the training of parameters associated with their respective HMM from a training corpus. The estimation of model parameters is made by applying the Baum-Welch optimal algorithm until convergence and reestimate probabilities of emission and transition.

\section{- Recognition}

Recognition is achieved by the Viterbi algorithm, it is used to find the most likely sequence of hidden states, called the Viterbi path, which results in a sequence of observed events.

The different steps of the recognition algorithm construction of HMM under platform HTK are illustrated in Figure 3.

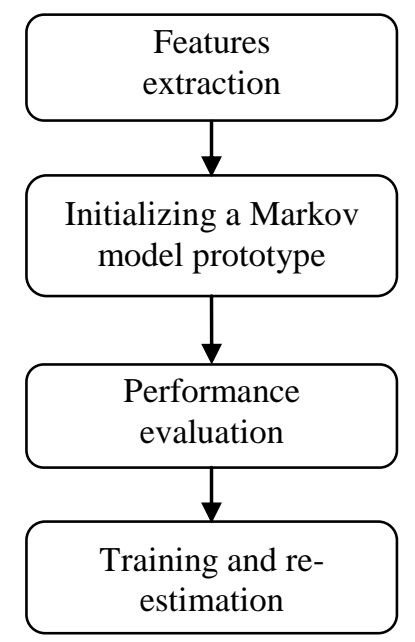

Fig 3. Experimental configuration of HMM model under HTK platform.

\section{EXPERIMENTAL RESULTS}

The Electrocardiogram records from the MIT $/ \mathrm{BIH}$ database [8] were used in this study. We used only 18 healthy signals from different individuals. The duration of each recording is 30 minutes and the sampling frequency is $360 \mathrm{~Hz}$. An extraction of the the Cepstral coefficients from each heart beat was made and the LFCC-RASTAMAT package [15] was used. 


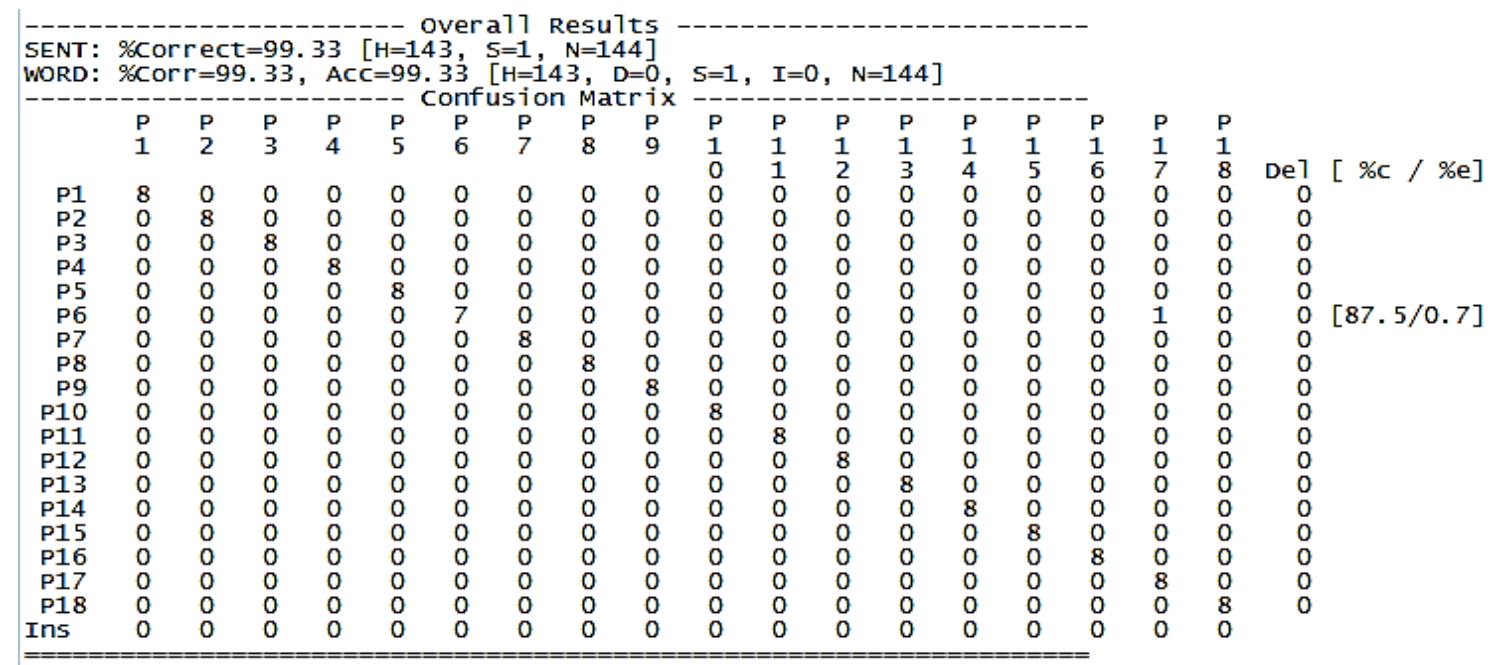

Fig 4. Confusion Matrix.

TABLE I. RECOGNITION RATE FOR DIFFERENT NUMBER OF COEFFICIENTS

\begin{tabular}{|l|l|l|l|l|l|l|l|l|l|l|l|l|}
\cline { 2 - 10 } \multicolumn{1}{c|}{} & \multicolumn{10}{c|}{ Number of coefficients } \\
\cline { 2 - 11 } & 12 & 14 & 16 & 18 & 20 & 22 & 24 & 26 & 28 & 30 & 32 & 34 \\
\hline $\begin{array}{l}\text { Recognition } \\
\text { rates }\end{array}$ & $88.5 \%$ & $89.17 \%$ & $94.14 \%$ & $95.50 \%$ & $96.12 \%$ & $96.33 \%$ & $96.05 \%$ & $97.25 \%$ & $97.33 \%$ & $98.61 \%$ & $\mathbf{9 9 . 3 1 \%}$ & $98.05 \%$ \\
\hline
\end{tabular}

TABLE II. RECOGNITION RATE FOR DIFFERENT NUMBER OF FREQUENCY BANDS WITH 5 STATES AND 1 GAUSSIAN.

\begin{tabular}{|l|l|l|l|l|l|l|l|l|l|l|l|l|}
\cline { 2 - 9 } \multicolumn{1}{c|}{} & \multicolumn{10}{c|}{ Number of frequency bands } \\
\cline { 2 - 7 } & 12 & 14 & 16 & 18 & 20 & 22 & 24 & 26 & 28 & 30 & 32 & 34 \\
\hline $\begin{array}{l}\text { Recognition } \\
\text { rates }\end{array}$ & $94.4 \%$ & $96.53 \%$ & $97.05 \%$ & $97.23 \%$ & $98.17 \%$ & $98.33 \%$ & $\mathbf{9 9 . 3 1 \%}$ & $\mathbf{9 9 . 3 1 \%}$ & $\mathbf{9 9 . 3 1 \%}$ & $\mathbf{9 9 . 3 1 \%}$ & $\mathbf{9 9 . 3 1 \%}$ & $\mathbf{9 9 . 3 1 \%}$ \\
\hline
\end{tabular}

In order to find the best results, the number of coefficients and the number of bank filter were varied from 12 to 34 .

Table 1 presented the variation of human recognition rate for different number of coefficients with one gaussian and 5 states. It is to be noticed that 32 coefficients gave a better result. Then, the number of bank filter by window was varied for 32 coefficients. One can note that the rate became constant from 24 filter banks. Then it decreased in thirty the fourth bank filter.Table 2 illustrated those results.

Thus, the best configuration of cepstral features extraction used in this paper is 32 cepstral coefficients, 24 frequency bands and the width of window is 0.5 seconds with $50 \%$ overlap.

Then, the cepstral coefficients using HMM were classified. For this, an approach two-thirds one third was used. That is to say, 20 minutes of ECG records were used for training and the last 10 minutes for testing.
Assessments for improved model topology of classifier, the number of Gaussian were varied between 1 to 5 and the number of state from 5 to 7 . The best rates are obtained for HMM topology with 1 Gaussian and 5 states which is the Bakis model.

Table 3 presented the recognition rates for 32 coefficients with different states and several number Gaussians. The best rate reached $99.31 \%$ for 1 Gaussian and 5 states. The more the number of Gaussians and number of states increase, the more the rate decreases.

TABLE III. RECOGNITION RATES FOR DIFFERENT STATES WITH SEVERAL GAUSSIAN NUMBERS PER STATES.

\begin{tabular}{|l|l|l|l|}
\cline { 2 - 4 } \multicolumn{1}{c|}{} & 1 Gaussian & 3 Gaussians & 5 Gaussians \\
\hline 5 States & $\mathbf{9 9 . 3 1 \%}$ & $95.33 \%$ & $88.67 \%$ \\
\hline 6 States & $95.50 \%$ & $89.17 \%$ & $88.33 \%$ \\
\hline 7 States & $92.83 \%$ & $90 \%$ & $87.50 \%$ \\
\hline
\end{tabular}


In order to improve the results, dynamic features which are first order delta $(\Delta)$ and energy (E) were added to the coefficients. The experimental results are illustrated in Table2 which reports the recognition rates obtained for each case.

As it can be seen, the best performance of our system has been obtained by 32 cepstral coefficients plus its Energy that make a 33 dimensional feature vector and the result is reaching $99.33 \%$.

TABLE IV. RECOGNITION RATE FOR DIFFERENT NUMBER OF FREQUENCY BANDS WITH 5 STATES AND 1 GAUSSIAN.

\begin{tabular}{|l|l|l|l|}
\hline LFCC & LFCC+ E & LFCC+ $+\Delta$ & LFCC+E+ $+\Delta$ \\
\hline $93.31 \%$ & $\mathbf{9 9 . 3 3 \%}$ & $92.50 \%$ & $91.67 \%$ \\
\hline
\end{tabular}

In previous work [2], the temporal parameters which are morphological descriptors and Hermite Polynomials Expansion Coefficients (HPEc) were classified with Hidden Model Markov (HMM).

According to the obtained results concerning this paper, the study concludes that the cepstral information in the homomorphic domain has given a human recognition rate that is equal $99.33 \%$ which remains slightly higher than that temporal information being $99.02 \%$.

\section{CONCLUSION}

We presented in this paper, a new personal identification method which used the cepstral information in the homomorphic domain. The RASTAMAT Package was used for extracting of the Lineair Frequency Cepstral Coefiicients.

Electrocardiogram (ECG) data for this investigation was obtained from MIT BIH database. Using this method, $99.33 \%$ human recognition rate was achieved for 18 healthy subjects. An interesting rate for identifying normal people is obtained by adding Energy to cepstral coefficients. That's why this study could be considered as a motivation towards the use of the cepstral information in the Human recognition system.

For future identification systems, the cepstral information with other parameters in time domain can be combined and multimodal biometrics can be used by associating the Electrocardiogram signal with other biometric parameters.

\section{REFERENCES}

[1] A. K. Jain, A. Ross, and S. Prabhakar, "An introduction to biometric systems,"IEEE Transactions on 188 Circuit and Systems for Video Technology, vol. 14, no. 1, pp.4-20, 2004.

[2] E.Rabhi, and Z. Lachiri," Biometric Personal Identification System using the ECG Signal," Computing in Cardiology Conference (CinC), vol.40,pp.507-510,2013.

[3] G.D.Clifford," A Novel Framework for Signal Representation and Source Separation,"Applications to Filtering and Segmentation of Biosignals, Journal of Biological Systems, Vol. 14, No. 2, pp. 169-183 June 2006.

[4] L. Biel, O. Pettersson, L. Philipson, and P. Wide, "ECG analysis: a new approach in human identification," IEEE Transactions on Instrumentation and Measurement, vol. 50,no. 3, pp. 808-812, 2001.

[5] L.R. Rabiner," A tutorial on hidden markov models and selected applications in speech recognition," Proceedings of the IEEE,vol.7,pp.257-286,1989.

[6] M.Li, S.Narayanan," Robust ECG Biometrics by Fusing Temporal and Cepstral Information," Pattern Recognition (ICPR), pp.1326-1329, 2010.

[7] M.Li, V. Rozgic, G.Thatte, S.Lee, A.Emken, M.Annavaram, U. Mitra, D.Spruijt-Metz and S.Narayanan, "Multimodal Physical Activity Recognition by Fusing Temporal and Cepstral Information," IEEE Transactions on Neural Systems \& Rehabilitation Engineering, vol 18, issue4, August, 2010.

[8] MIT-BIH Normal Sinus Rhythm Database, available online: http://www.physionet.org/physiobank/database.

[9] N. Belgacem, F.B. REGUIG and A.NAIT-ALI, "Person Identification System Based on Electrocardiogram Signal Using LabVIEW, " International Journal on Computer Science and Engineering (IJCSE), Vol. 4 No. 06 June 2012.

[10] S. A. Israel, J. M. Irvine, A. Cheng, M. D. Wiederhold, and B. K. Wiederhold, "ECG to identify individuals," Pattern Recognition, vol. 38, no. 1, pp. 133-142, 2005.

[11] S.Young," The HTK Hidden Markov Model Toolkit,” Design and Philosophy. Cambridge University Engineering Department - Technical report 152, 1994.

[12] S.Young, G. .Evermann, D. Kershaw, G. Moore, J. Odell, D. Ollason, D. Povey, V. Valtchev, and P. Woodland, The HTK Book 3.2, Cambridge University Engineering Department - Speech Group, 2001-2006.

[13] T. W. Shen, W. J. Tompkins, and Y. H. Hu, "One-lead ECG for identity verifica-tion," in Proc. of the 2nd Conf. of the IEEE Eng. in Med. and Bio. Society and the Biomed. Eng. Society, vol. 1, pp. 62-63,2002.

[14] X. Zhou, D. Garcia-Romero, R. Duraiswami, C. Espy-Wilson, S. Shamma, "Linear versus Mel- Frequency cepstral coefficients for speaker recognition", ASRU 2011 (IEEE Automatic Speech Recognition and Understanding Workshop).

[15] X. Zhou (2011), LFCC- RASTA in Matlab, available online: http://terpconnect.umd.edu/ Zxinhui/ 